\title{
A Complete Inventory of Long-Chain Polyunsaturated Fatty Acids Biosynthesis Pathway Enzymes in the Miniaturized Cyprinid Paedocypris Micromegethes
}

\section{Ka-Kei Sam}

Universiti Sains Malaysia

Nyok-Sean Lau

Universiti Sains Malaysia

Meng-Kiat Kuah

Universiti Sains Malaysia

Engkamat anak Lading

Sarawak Forestry Department

Alexander Chong Shu-Chien ( $\square$ alex@usm.my )

Universiti Sains Malaysia https://orcid.org/0000-0003-3014-442X

\section{Research Article}

Keywords: Paedocypris micromegethes, miniaturized fish, long-chain polyunsaturated fatty acids, Fads, Elovl, tropical peat swamp

Posted Date: January 18th, 2022

DOI: https://doi.org/10.21203/rs.3.rs-1255382/v1

License: (c) (i) This work is licensed under a Creative Commons Attribution 4.0 International License.

Read Full License 


\section{Abstract}

The capacity for long-chain polyunsaturated fatty acid (LC-PUFA) biosynthesis activity in a species depends on the enzymatic activities of fatty acyl desaturase (Fads) and elongation of very long-chain fatty acid (Elovl). The miniaturized fish Paedocypris micromegethes is a developmentally truncated cyprinid living in highly acidic water conditions in tropical peat swamps. The capacity for LC-PUFA biosynthesis in this species, which has a reduced genome size, is unknown. A high-quality de novo transcriptome assembly enabled the identification of a putative Fads2 and four Elovl. The Fads2 was verified as a $P$. micromegethes Fads 2 ortholog with in vitro $\Delta 5$ and $\Delta 6$ activities. The Elovl sequences were established as an Elovl5, Elovl2, and two Elovl4 paralogs, namely Elovl4a and Elovl4b. These Elovl enzymes, mainly Elovl5 and Elovl2, fulfill the necessary C18, C20, and C22 PUFA elongation steps for LCPUFA biosynthesis. Collectively, these results validate the presence of a complete repertoire of LC-PUFA biosynthesis enzymes in a tropical miniatured freshwater fish.

\section{Introduction}

Long-chain polyunsaturated fatty acids (LC-PUFAs), consisting of eicosapentaenoic acid (EPA; $20: 5 n-3)$, docosahexaenoic acid (DHA; 22:6n-3), and arachidonic acid (ARA; 20:4n-6), are required for proper cellular membrane structure, signaling, gene regulation, and energy (Jump 2002). LC-PUFAs can be synthesized from C18 PUFA precursors de novo through the concerted action of enzymes known as fatty acyl desaturases (Fads) and elongation of very long-chain fatty acid (Elovl) proteins (Sprecher 1981). Fads catalytic activity involves introducing a double bond at a specific position of the fatty acyl backbone while the elongation of the fatty acyl chain with two carbon units is by Elovl catalyzing the ratelimiting condensation step (Jakobsson et al. 2006). In the conventional biosynthesis pathway, linolenic acid (ALA, 18:3n-3) and linoleic acid (LA, 18:2n-6) are converted to EPA and ARA, respectively, through two possible paths, either utilizing a $\Delta 6$ or $\Delta 8$ desaturation route (Park et al. 2009). Subsequent conversion of EPA to DHA could occur through the 'Sprecher' pathway where EPA is first converted to $24: 5 n-3$, followed by a $\Delta 6$ desaturation and finally a $\beta$-oxidation step (Sprecher et al. 1995), or through a more direct route, which involves the elongation of EPA to docosapentaenoic acid (DPA, 22:5n-3), followed by a direct $\Delta 4$ desaturation to DHA (Li et al. 2010). To date, two Fads with different desaturation roles within the LCPUFA biosynthesis pathway, namely Fads1 and Fads2, have been identified in vertebrates (Castro et al. 2012). As for Elovl, four Elovl families, namely Elovl2, 4, 5, and 8 have been identified to have PUFA elongation activities (Jakobsson et al. 2006; Sun et al. 2021).

Terrestrial consumers rely almost exclusively on the export of LC-PUFAs from various aquatic ecosystems (Gladyshev et al. 2009). This is due to the existence of various producers and primary consumers with de novo LC-PUFA biosynthesis capacities within the aquatic food web (Kabeya et al. 2018; Twining et al. 2021). Teleost also possesses different capacity for LC-PUFA biosynthesis, depending of the catalog of Fads and Elovl within the species. Findings so far have proposed the influence of species habitat, trophic level, evolutionary history, and taxonomy position on the LC-PUFA biosynthesis capacity (Garrido et al. 2019; Matsushita et al. 2020; Xie et al. 2021). Multiple works on the Fads/Elovl enzymes in species from 
key phylogenetic lineages within the chordates have shown the contribution of whole genome duplication, incidences of neo-functionalizations, sub-funtionalizations and gene loss in diversifying the number and function of Fads and Elovl in different species (Castro et al. 2016; Monroig et al. 2016; Xie et al. 2021).

The order Cypriniformes is represented by over 3,000 species, with many members of this order being major aquaculture food and ornamental fish species. Cyprinids have a widespread distribution, with natural freshwater populations in all continents except Oceania, Antarctic and South America (Berra 2001). While the major carp species are large-sized, cyprinids also include a group of dwarf-like fish, which underwent a dramatic reduction of body size, with a maturation size of $\leq 20 \mathrm{~mm}$ standard length (Weitzman and Vari 1988). This event, known as miniaturization, is intriguingly common in cyprinids, with over 20 species in South, Southeast Asia and Africa (Kottelat et al. 2006; Ruber et al. 2007). An extreme example is found in the genus Paedocypris, represented by $P$. progenetica, $P$. micromegethes and $P$. carbunculus, which are recognized as the world's smallest fish and vertebrate (Britz and Kottelat 2008; Kottelat et al. 2006; Ruber et al. 2007). Paedocypris is confined to Southeast Asia peat swamp forests, inhabiting the highly fragmented waterlogged swamps with highly acidic and tannin-rich waters low in nutrients and dissolved oxygen (Kottelat et al. 2006).

To date, several cyprinid species have been validated to have all the necessary Fads and Elovl for LCPUFA biosynthesis, including the zebrafish (Agaba et al. 2004; Hastings et al. 2001; Monroig et al. 2010a) silver barb (Janaranjani et al. 2018; Janaranjani and Shu-Chien 2020), tench (Garrido et al. 2020) and giant mahseer (Sam et al. 2021). The draft genome assemblies of $P$. micromegethes and $P$. carbunculus have reported reduced genome size (Malmstrøm et al. 2018). In addition, very little is known about the production and transfer of PUFA in peat swamp waters. We report here the cloning and functional characterization of all the hypothetically necessary Fads and Elovl required for a functional LC-PUFA biosynthesis pathway in P. micromegethes. Our results suggest the retaining of a complete LC-PUFA biosynthesis pathway in this species, which most likely ensure successful colonization of the peat swamp environment.

\section{Materials And Methods}

Sample collection, total RNA extraction and sequencing

Adult $P$. micromegethes was obtained from a peat swamp forest in Matang, Sarawak, Malaysia $\left(01^{\circ}\right.$ $34.78067^{\prime} \mathrm{N}, 110^{\circ} 16.04233^{\prime} \mathrm{E}$ ) from December-January 2019 under the permit 63/2018 under the reference of (114)JHS/NCCD/600-7/2/107. Collected samples were preserved in RNAlater® (Thermo Scientific, USA) and stored at $-80^{\circ} \mathrm{C}$ before extraction. All experimental procedures were performed in accordance with the USM Institutional Animal Care and Use Committee guidelines under the approval code USM/IACUC/2019/(117)(988). The RNA extraction was carried out using RNeasy Mini Kit (Qiagen, USA) with DNase treatment, according to the manufacturer instruction. The quantity of RNA was measured using the Qubit ${ }^{\circledR}$ RNA BR Assay Kit on Qubit ${ }^{\circledR}$ 2.0 Fluorometer (Invitrogen, Life Technologies). 
RNA integrity was validated by Agilent 2100 Bioanalyzer Nano kit (Santa Clara, CA). Total RNA obtained from the three biological replicates was sent for library construction, followed by RNA sequencing using the HiSeq $X^{\text {TM }}$ Ten platform (Illumina, USA) with paired-end $150 \mathrm{bp}$.

De novo assembly, filtering and annotation of RNA-seq data

Nearly 235 million raw reads were generated from the three independent libraries. The paired-end data was processed for removal of adapter, low-quality sequences (quality score $<5$; reads $<36 \mathrm{bp}$ ) using TrimGalore (https://www.bioinformatics.babraham.ac.uk/projects/trim_galore/). FastQC (https://www.bioinformatics.babraham.ac.uk/projects/fastqc/) was used to assess sequence quality. De novo transcriptome assembly was done with Trinity (Grabherr et al. 2011) software with parameters '-SS_lib_type RF --seqType fq -left <input_file> -right <input_file> --max_memory 500G --CPU 48 > run.log $2>\& 1$ '. A perl script, inside the Trinity package (TrinityStats.pl) was used to compute the assembly statistic. To obtain the non-redundant transcripts, clustering analysis was performed for redundancy reduction using the CD-Hit program v4.8.1 (Li and Godzik 2006) with an identity threshold of $90 \%$. Benchmarking Universal Single-Copy Orthologs (BUSCOs) was applied to access the transcriptome completeness using the Actinopterygii dataset (Kriventseva et al. 2015). Coding regions of the nonredundant assembled transcripts were predicted with the TransDecoder v5.5.0

(https://github.com/TransDecoder/TransDecoder) pipeline using a minimum cut-off of 200 amino acids (aa) and open reading frames (ORFs). Functional annotation of the generated sequences was performed through BLASTp homology search on Genbank Nr, SwissProt, KEGG, KOG, and Pfam databases with Evalue cut-off of $1 \cdot 10^{-5}$. The BLAST results against the $\mathrm{Nr}$ database were processed through Blast2go to retrieve the Gene Ontology (GO) annotation (Conesa et al. 2005). KEGG pathway analysis was conducted using the online KEGG Automatic Annotation Server (KAAS) https://www.genome.jp/kegg/kaas/ with the bi-directional best hit (BBH) method for KEGG orthology (KO) assignment.

Identification and phylogenetic analysis of Fads and Elovl

The putative sequences of known LC-PUFA biosynthesis Fads and Elovl from cyprinid species (fads2, elov/5, elov/2, elov/4a, and elov/4b) were identified and extracted from the transcriptome assembly through tblastn search with Fads and Elovl sequences as queries. Online InterProScan tool was used to infer the protein domains (http://www.ebi.ac.uk/Tools/pfa/iprscan/). A putative full-length Fads transcript was mined utilizing two Pfam domains namely N-terminal Cyt-b5 (PF00173) and FA_desaturase (PF00487), and three histidine motifs (HXXXH, HXXXHH and QXXHH). For Elovl orthologs, full-length transcripts consisting of a Pfam domain ELO (PF01151) and a histidine motif (HXXHH) were discovered from the assembly. Multiple sequence alignment of the discovered protein sequences with orthologs from various vertebrates was done using Clustal Omega program (https://www.ebi.ac.uk/Tools/msa/clustalo/).

For phylogenetic analysis, protein sequences were aligned with MAFFT v7.475 using the L-INS-i method. The alignment was then used to determine the best-fit substitution model using ModelFinder based on 
Akaike information criterion (AIC). Maximum likelihood (ML) tree were generated for Fads and Elovls respectively, using the IQ-TREE v1.6.12 (Nguyen et al. 2014) software with JTT+G4+I substitution model and 1,000 bootstraps. The consensus trees of the ML phylogenies were visualized through FigTree v1.4.2.

cDNA cloning of the fads2, elov/5, elov/2, elov/4a, and elov/4b

Total RNA was extracted from three adult fishes as described in 2.1. Subsequently, cDNA was synthesized from $3 \mu \mathrm{g}$ of the total RNA with Oligo $\mathrm{d}(\mathrm{T})_{20}$ primer using high performance SuperScript IV Reverse Transcription kit (Invitrogen). Gene-specific primers for amplification of the fads2, elov/5, elov/2, elov/4a, and elov/4b sequences were designed based on the ORF sequences generated from the TransDecoder (Table S1). PCR was performed using KAPA HiFi HotStart polymerase (KAPA Biosystems, USA) with the following settings: $95^{\circ} \mathrm{C}$ for $3 \mathrm{~min}, 35$ cycles of $98^{\circ} \mathrm{C}$ for $20 \mathrm{~s}, 60^{\circ} \mathrm{C}$ for $30 \mathrm{~s}$ and $72^{\circ} \mathrm{C}$ for 1 min, and $72^{\circ} \mathrm{C}$ for $1 \mathrm{~min}$. The final PCR products were purified and digested with corresponding restriction enzymes and cloned into yeast expression vector pYES2 (Invitrogen, USA).

Functional characterization of the fads2, elov/5, elov/2, elov/4a, and elov/4b

Purified plasmid constructs pYES2_fads2, pYES2_elovl5, pYES2_elov/2, pYES2_elov/4a, and pYES2_elovl4b, together with an empty pYES2_ without ORF were transformed into the yeast Saccharomyces cerevisiae (strain INVSc1), respectively. Transformation and culture of yeast were as previously (Kuah et al. 2015). For characterization of fads $2 \Delta$ desaturation capacity, transgenic yeast expressing the ORF was incubated in the presence of the following fatty acid (FA) substrates: $18: 3 n-3$, 18:2n-6, 24:5n-3, and 24:4n-6 for $\Delta 6,20: 3 n-3$ and $20: 2 n-6$ for $\Delta 8,20: 4 n-3$ and $20: 3 n-6$ for $\Delta 5$ and 22:5n-3 and 22:4n-6 for $\Delta 4$. For elovl, 18:3n-3, 18:2n-6, 18:4n-3, 18:3n-6, 20:5n-3, 20:4n-6, 22:5n-3, and 22:4n-6 substrates were assayed. In addition, $\mathrm{C} 24: 0$ and C22:6n-3 substrates were also assessed for elov/4a and elov/4b. The final FA concentration added to the yeast culture were $0.5 \mathrm{mM}$ (C18), $0.75 \mathrm{mM}$ (C20), $1.0 \mathrm{mM}$ (C22), and $1.25 \mathrm{mM}$ (C24), respectively. Each assay was repeated thrice with replicate recombinants.

Fatty acid analysis

After two days of incubation with FA substrates $\left(30^{\circ} \mathrm{C}, 250 \mathrm{rpm}\right)$, yeast was harvested, followed by total of lipid extraction in chloroform/methanol $(2: 1, \mathrm{v} / \mathrm{v})$. Fatty acid methyl ester (FAME) was extracted and purified as described previously (Kuah et al. 2016). The derived FAME was characterized in a gas chromatography-mass spectrometry (GC-MS) QP2010 (Shimadzu, USA), equipped with a Supelco 2380 high-polarity fused-silica capillary column (30 m length, $0.25 \mathrm{~mm}$ inner diameter, $0.20 \mu \mathrm{m}$ film thickness: SGE, USA). Helium was applied as carrier gas, with the oven temperature maintained at $80^{\circ} \mathrm{C}$, followed by an increase to $260^{\circ} \mathrm{C}$ at a rate of $2^{\circ} \mathrm{C} / \mathrm{min}$. The synthesized FA products were identified and confirmed based on retention time and the mass spectrometry NIST08s mass library. The desaturation or elongation conversion efficiency was calculated as [individual product area / (all products areas + substrate area)] . 100. 
The FA composition of whole body P. micromegethes was determined after total lipid extraction and FAME extraction as mentioned above. The FA composition was calculated as [individual peak area/total peak area] $x 100$. All results are represented as mean \pm SEM from the three independent samples.

\section{Result}

De novo transcriptome assembly and annotation

From the de novo transcriptome assembly, a total of 210,210 transcripts with a mean length of $1,021 \mathrm{bp}$ and N50 of 2,350 bp were obtained. The assembly statistics of the $P$. micromegethes are presented in Table 1. Analysis of the BUSCOs (Benchmarking Universal Single Copy Orthologs) against Actinopterygii gene set on the non-redundant transcripts revealed a high percentage of complete orthologues (93.4\%), either as single or duplicated sequences (Fig. S1a). The protein coding sequences (cds) within the transcripts were predicted using TransDecoder. Four ORF categories, which are complete ORF $(44,083), 5^{\prime}$ partial ORF $(10,602), 3$ ' partial ORF $(3,975)$, and internal $\operatorname{ORF}(2,326)$ were obtained (Fig. S1b). Based on the homology search, $87.98 \%, 82.72 \%, 86.82 \%, 81.64 \%$, and $74.63 \%$ of the peptide sequences showed significant matched to the Nr, SwissProt, KEGG, KOG, and Pfam databases, respectively (Fig. S1c). Another $71.11 \%$ of sequences were assigned with $\mathrm{GO}$ term consisting of three independent categories: biological process, molecular and cellular functions (Fig. S2). The highest $P$. micromegethes transcripts assigned into the biological process category belongs to cellular process, biological regulation, and response to stimulus. As for molecular function category, the top assigned transcripts were grouped into catalytic, transcription regulator and molecular transducer activities while for cellular function category, highest transcripts were assigned to cellular anatomical entity and protein-containing complex. KEGG Automatic Annotation Server (KAAS) assigned KO IDs to 22,003 of the transcripts, with 8,673 assigned as complete, with involvement in 403 pathways (Table S2). 
Table 1

General statistics for the de novo assembly Trinity of $P$. micromegethes

\begin{tabular}{|ll|}
\hline Assembly characteristics & Value \\
\hline Before filtering & $235,289,694$ \\
\hline Raw reads (PE) & \\
\hline Total assembled bases (bp) & $518,275,680$ \\
\hline Total assembled contigs & 388,294 \\
\hline Average contig length (bp) & 1,334 \\
\hline Median contig length (bp) & 599 \\
\hline Contig N50 length (bp) & 2,715 \\
Contig N90 length (bp) & 483 \\
GC content (\%) & 48.07 \\
After filtering* & $214,789,660$ \\
Total assembled bases (bp) & 210,210 \\
\hline Total assembled contigs & 1,021 \\
Average contig length (bp) & 413 \\
Median contig length (bp) & 2,350 \\
Contig N50 length (bp) & 337 \\
Contig N90 length (bp) & 46.90 \\
GC content (\%) & \\
\hline * Redundant transcripts were eliminated using CD-HIT. \\
\hline
\end{tabular}

Sequence and phylogenetic analyses of the Fads and Elovl enzymes in P. micromegethes

A total of five selected transcripts, corresponding to the Fads and Elovl involved in LC-PUFA biosynthesis were mined from the $P$. micromegethes transcriptome. This includes a putative fads 2 and four elovls, including the elov/5, elov/2 and two elov/4 paralogs, elov/4a and elov/4b (Table 2). The ORFs length of the P. micromegethes fads2, elov/5, elov/2, elov/4a, and elov/4b transcripts are 1,335 bp, 810 bp, 849 bp, 933 bp, and 903 bp, respectively, encoding the protein sequences of 445 aa, 270 aa, 283 aa, 311 aa, and 301 aa. 
Table 2

List mined putative Fads and Elovl transcripts involved in the biosynthesis of polyunsaturated fatty acids for $P$. micromegethes

\begin{tabular}{|ll|}
\hline Annotated complete sequence_ID & Sequence description \\
\hline TRINITY_DN36236_c1_g1_i1.p1 & Delta-6 desaturase (Fads2) \\
\hline TRINITY_DN38107_c1_g5_i10.p1 & Elongation of very long chain fatty acids 5 (Elovl5) \\
\hline TRINITY_DN33464_c1_g2_i2.p1 & Elongation of very long chain fatty acids 2 (Elovl2) \\
\hline TRINITY_DN30298_c0_g2_i1.p1 & Elongation of very long chain fatty acids 4a (Elovl4a) \\
\hline TRINITY_DN36298_c3_g2_i1.p1 & Elongation of very long chain fatty acids 4b (Elovl4b) \\
\hline
\end{tabular}

The BLASTp searches showed that the $P$. micromegethes Fads2 is highly identical (>85\%) with functionally characterized orthologs from cyprinids, including Tinca tinca (QIA97820), Tor tambroides (AZL94116), Danio rerio (Q9DEX7), and Barbonymus gonionotus (AXF92413). Alignment of the $P$. micromegethes putative Fads2 with six functional characterized Fads protein sequences from Homo sapiens, Clarias gariepinus, B. gonionotus, D. rerio, Siganus canaliculatus $(\Delta 4)$, and $S$. canaliculatus $(\Delta 6 /$ $\Delta 5$ ) showed typical microsomal fatty acyl front-end Fads sequence features (Fig. 1). This includes a $\mathrm{N}$ terminal cytochrome $b_{5}$-like domain, a heme binding motif HPGG, three conserved histidine boxes ( $H X X X H, H X X H H$ and QXXHH), and four putative transmembrane domains. A region (FQFQ) speculated to be important for the regioselectivity of Fads 2 is also present. The tree topology of Fads protein sequences showed two well-supported distinct clades, Fads1 and Fads2, with the $P$. micromegethes ortholog placed within the latter group (Fig. 2). Within the Fads2 clade, the various Cypriniformes members are grouped together with orthologs from other Otomorpha fish, including Siluriformes and Characiformes. The other Fads2 clade is made of a branch containing Salmoniformes and a larger branch comprises of Fads2 from various Acantomorphan.

As for Elovls, BLASTp analysis on Elovl5 matched this sequence (79.0-77.6\%) with functionally characterized Elovl5 of other cyprinids including T. tambroides, B. gonionotus and D. rerio. Likewise, the P. micromegethes Elovl2 is highly identical with B. gonionotus, T. tinca and T. tambroides (86.8-84.8\%). The P. micromegethes Elovl4a and Elovl4b were 92.0-81.5\% similar with several teleost Elovl4 elongases. The alignment of all the above elongases (Fig. 3) showed typical characteristics of a microsomal fatty acyl elongase: four conserved motifs (KXXEXXDT, QXXFLHXXHH, NXXXHXXMYXYY, and TXXQXX), seven putative transmembrane domains, and a histidine rich box $(H X X H H)$. The putative endoplasmic reticulum (ER) retrieval signal with KXRXX in Elovl5, KXKRX in Elovl2 and $R(K) X K X X$ in Elovl4 were also detected at the carboxyl terminal. The ML tree topology showed the Elovl2 and Elovl5 orthologs sharing a common ancestor whereas all Elovl4 orthologs are in a separate cluster (Fig. 4). The Elovl4a and Elovl4b isoforms are separated into two respective clades.

Functional characterization of $P$. micromegethes Fads2 desaturase and Elovl elongases 
The FA composition of the P. micromegethes fads 2 ORF-inserted S. cerevisiae yeast indicates $\Delta 6$ desaturation activity towards C18 PUFA substrates (Table 3 ). A low and probably negligible $\Delta 8$ activity towards 20:3n-3 was detected. Furthermore, $P$. micromegethes fads 2 was able to catalyze the biosynthesis of EPA and ARA through $\triangle 5$ desaturation towards C20:4n-3 and C20:3n-6 substrates, respectively. Meanwhile, a single peak corresponding to the non-methylene interrupted (NMI) product $(\Delta 5,11,14,1720: 4)$ was also detected. Incubation of the transformed yeast with either of the C22 PUFA substrates did not yield any products, which implies a lack of $\Delta 4$ desaturation. Incubation with C24:4n-6 or $\mathrm{C} 24: 5 \mathrm{n}-3$ resulted in their respective desaturation products, which suggest a role in this Fads 2 in the 'Sprecher' pathway for the biosynthesis of DHA. Overall, the results indicate that the P. micromegethes Fads is a Fads2 with bifunctional $\Delta 6 / \Delta 5$ activities.

Table 3

Functional characterization of $P$. micromegethes Fads2 through heterologous expression of the respective transcript ORF in yeast (Saccharomyces cerevisiae), followed by incubation with PUFA substrate to assay the desaturation rate leading to PUFA product

\begin{tabular}{|c|c|c|c|}
\hline PUFA Substrate & PUFA Product & Conversion rate (\%) & Activity \\
\hline & & Fads2 & \\
\hline $18: 3 n-3$ & $18: 4 n-3$ & $26.8 \pm 1.7$ & $\Delta 6$ \\
\hline $18: 2 n-6$ & $18: 3 n-6$ & $13.8 \pm 0.4$ & $\Delta 6$ \\
\hline $20: 3 n-3^{*}$ & $20: 4 n-3$ & $0.9 \pm 0.1$ & $\Delta 8$ \\
\hline $20: 2 n-6$ & $20: 3 n-6$ & 0 & $\Delta 8$ \\
\hline $20: 3 n-3^{a}$ & $\Delta 5,11,14,1720: 4$ & $4.8 \pm 0.5$ & $\Delta 5$ \\
\hline $20: 4 n-3$ & $20: 5 n-3$ & $18.6 \pm 1.0$ & $\Delta 5$ \\
\hline $20: 3 n-6$ & $20: 4 n-6$ & $7.34 \pm 1.0$ & $\Delta 5$ \\
\hline $22: 5 n-3$ & $22: 6 n-3$ & 0 & $\Delta 4$ \\
\hline $22: 4 n-6$ & $22: 5 n-6$ & 0 & $\Delta 4$ \\
\hline $24: 5 n-3$ & $24: 6 n-3$ & $7.5 \pm 0.6$ & $\Delta 6$ \\
\hline $24: 4 n-6$ & $24: 5 n-6$ & $10.0 \pm 2.0$ & $\Delta 6$ \\
\hline
\end{tabular}

Results are expressed as the percentage of the PUFA substrate converted to PUFA product with the following calculation: [individual product area / (all products area + substrate area)] $\times 100$. Value is presented as the mean \pm SEM $(n=3)$.

*Conversion of $20: 3 n-3$ involved step wise reactions of $\Delta 8$ desaturation, followed by a $\Delta 5$ desaturation of $20: 4 n-3$ to EPA.

** 20:3n-3 was also converted to non-methylene interrupted fatty acid, $\Delta 5,11,14,1720: 4$.

ND = not detected; PUFA= Polyunsaturated fatty acid 
Transgenic yeast harboring the ORF of $P$. micromegethes elov/5 was able to elongate $\mathrm{C} 18$ and C20 PUFA substrates, with highest activities $\mathrm{C} 18: 4 \mathrm{n}-3$ and C18:3n-6 substrates. There was no elongation of $\mathrm{C} 22$ substrates, implying the $P$. micromegethes Elovl5 is a C18-C20 PUFA elongase (Table 4). In comparison, the P. micromegethes elov/2 ORF catalyzed the elongation of all tested C18, C20 and C22 PUFA substrates. Notably, this ortholog exhibits elongation of 22:5n-3, and 22:4n-6 substrates to $C_{24}$ product, with the former a significant activity in the production of DHA. As for Elovl4, both the $P$. micromegethes Elovl4 paralogs exhibited elongation capacity toward the saturated fatty acid (SFA) C24:0, with detection of very-long-chain saturated fatty acid (VLC-SFA) elongated products up to C30:0 (Table 5). There was also elongation of C18 and C20 PUFA substrates, and low elongation of C22 PUFA. Between the two isoforms, Elovl4a displayed higher elongation activities towards C18 PUFA. In contrast, the Elovl4b showed higher activities towards the $\mathrm{C} 20$ substrates, with further elongation to C36 PUFA substrates. All chromatograms of the yeast FA profiles are presented in supplementary section (Fig. S3-S7). 
Table 4

Functional characterization of $P$. micromegethes Elovl5 and Elovl2 through heterologous expression of the respective transcript ORF in yeast (Saccharomyces cerevisiae), followed by incubation with PUFA substrate to assay the elongation rate leading to PUFA product

\section{PUFA substrate PUFA product}

Conversation rate (\%)

Activity

\begin{tabular}{|c|c|c|c|c|c|}
\hline & & Pyes2 vector & Elovl5 & Elovl2 & \\
\hline \multirow[t]{4}{*}{$18: 3 n-3$} & $20: 3 n-3$ & $1.6 \pm 0.0$ & $6.8 \pm 0.1$ & $18.2 \pm 0.8$ & $\mathrm{C} 18 \rightarrow 20$ \\
\hline & $22: 3 n-3$ & ND & ND & $2.9 \pm 0.1$ & $\mathrm{C} 20 \rightarrow 22$ \\
\hline & $24: 3 n-3$ & ND & ND & ND & $\mathrm{C} 22 \rightarrow 24$ \\
\hline & Total & $1.6 \pm 0.0$ & $6.8 \pm 0.1$ & $21.1 \pm 0.9$ & \\
\hline \multirow[t]{4}{*}{$18: 2 n-6$} & $20: 2 n-6$ & $1.3 \pm 0.3$ & $2.3 \pm 0.0$ & $7.2 \pm 0.6$ & $\mathrm{C} 18 \rightarrow 20$ \\
\hline & $22: 2 n-6$ & $1.3 \pm 0.1$ & $0.6 \pm 0.1$ & $1.6 \pm 0.0$ & $\mathrm{C} 20 \rightarrow 22$ \\
\hline & $24: 2 n-6$ & ND & ND & ND & $\mathrm{C} 22 \rightarrow 24$ \\
\hline & Total & $2.6 \pm 0.4$ & $2.8 \pm 0.1$ & $8.8 \pm 0.6$ & \\
\hline \multirow[t]{4}{*}{$18: 4 n-3$} & $20: 4 n-3$ & $1.8 \pm 0.1$ & $39.4 \pm 3.0$ & $17.3 \pm 0.4$ & $\mathrm{C} 18 \rightarrow 20$ \\
\hline & $22: 4 n-3$ & ND & $0.3 \pm 0.0$ & $8.6 \pm 0.3$ & $\mathrm{C} 20 \rightarrow 22$ \\
\hline & $24: 4 n-3$ & ND & ND & $1.8 \pm 0.1$ & $\mathrm{C} 22 \rightarrow 24$ \\
\hline & Total & $1.8 \pm 0.1$ & $39.7 \pm 3.0$ & $27.8 \pm 0.6$ & \\
\hline \multirow[t]{4}{*}{$18: 3 n-6$} & $20: 3 n-6$ & $1.4 \pm 0.1$ & $32.0 \pm 2.2$ & $15.8 \pm 0.7$ & $\mathrm{C} 18 \rightarrow 20$ \\
\hline & $22: 3 n-6$ & ND & ND & $7.0 \pm 0.4$ & $\mathrm{C} 20 \rightarrow 22$ \\
\hline & $24: 3 n-6$ & ND & ND & $2.8 \pm 0.4$ & $\mathrm{C} 22 \rightarrow 24$ \\
\hline & Total & $1.4 \pm 0.1$ & $32.0 \pm 2.2$ & $25.5 \pm 1.0$ & \\
\hline \multirow[t]{3}{*}{$20: 5 n-3$} & $22: 5 n-3$ & ND & $12.5 \pm 2.6$ & $9.2 \pm 0.4$ & $\mathrm{C} 20 \rightarrow 22$ \\
\hline & $24: 5 n-3$ & ND & ND & $35.3 \pm 1.1$ & $\mathrm{C} 22 \rightarrow 24$ \\
\hline & Total & & $12.5 \pm 2.6$ & $44.5 \pm 1.4$ & \\
\hline $20: 4 n-6$ & $22: 4 n-6$ & ND & $4.4 \pm 0.7$ & $3.4 \pm 0.2$ & $\mathrm{C} 20 \rightarrow 22$ \\
\hline
\end{tabular}

Results are expressed as the percentage of the fatty acid substrate converted to elongated fatty acid products with the following calculation: [individual product area / (all products area + substrate area)] $\times 100$. Value is presented as the mean $\pm \operatorname{SEM}(n=3)$.

Total conversion is calculated based on summation of all of the elongated products.

ND = not detected; PUFA= Polyunsaturated fatty acid 


\begin{tabular}{|c|c|c|c|c|c|}
\hline \multirow[t]{2}{*}{ PUFA substrate } & \multicolumn{2}{|c|}{ PUFA product } & \multicolumn{2}{|c|}{ Conversation rate (\%) } & \multirow{2}{*}{$\begin{array}{l}\text { Activity } \\
\text { C22 } \rightarrow 24\end{array}$} \\
\hline & $24: 4 n-6$ & ND & ND & $20.4 \pm 1.8$ & \\
\hline & Total & & $4.4 \pm 0.7$ & $23.8 \pm 2.0$ & \\
\hline $22: 5 n-3$ & $24: 5 n-3$ & ND & ND & $10.8 \pm 0.5$ & $\mathrm{C} 22 \rightarrow 24$ \\
\hline $22: 4 n-6$ & $24: 4 n-6$ & ND & ND & $8.4 \pm 0.5$ & $\mathrm{C} 22 \rightarrow 24$ \\
\hline \multicolumn{6}{|c|}{$\begin{array}{l}\text { Results are expressed as the percentage of the fatty acid substrate converted to elongated fatty acid } \\
\text { products with the following calculation: [individual product area / (all products area + substrate area) } \\
\times 100 \text {. Value is presented as the mean } \pm \text { SEM }(n=3) \text {. }\end{array}$} \\
\hline \multicolumn{6}{|c|}{ Total conversion is calculated based on summation of all of the elongated products. } \\
\hline$N D=$ not detect $\epsilon$ & UFA= Poly & ted fa & & & \\
\hline
\end{tabular}


Table 5

Functional characterization of $P$. micromegethes Elovl4a and Elovl4b through heterologous expression of the respective transcript ORF in yeast (Saccharomyces cerevisiae), followed by incubation with SFA/PUFA substrate to assay the elongation rate leading to SFA/PUFA product

Substrate Product Conversion rate (\%)

Activity

Pyes2 vector Elovl4a Elovl4b

\section{SFA}

\begin{tabular}{rlllll}
$24: 0$ & $26: 0$ & $3.25 \pm 0.7$ & $5.2 \pm 0.3$ & $3.05 \pm 0.3$ & C24 $\rightarrow 26$ \\
\hline $28: 0$ & ND & $2.8 \pm 0.1$ & $1.6 \pm 0.3$ & C26 $\rightarrow 28$ \\
\hline $30: 0$ & ND & $1.9 \pm 0.1$ & $0.4 \pm 0.1$ & C28 $\rightarrow 30$ \\
$32: 0$ & ND & ND & ND & C30 $\rightarrow 32$
\end{tabular}

\section{PUFA}

\begin{tabular}{|c|c|c|c|c|c|}
\hline \multirow[t]{4}{*}{$18: 3 n-3$} & $20: 3 n-3$ & $1.3 \pm 0.3$ & $15.1 \pm 0.8$ & $8.3 \pm 0.1$ & $\mathrm{C} 18 \rightarrow 20$ \\
\hline & $22: 3 n-3$ & ND & $1.7 \pm 0.1$ & $1.0 \pm 0.1$ & $\mathrm{C} 20 \rightarrow 22$ \\
\hline & $24: 3 n-3$ & ND & ND & ND & $\mathrm{C} 22 \rightarrow 24$ \\
\hline & Total & $1.3 \pm 0.3$ & $16.9 \pm 0.8$ & $9.3 \pm 0.2$ & \\
\hline \multirow[t]{4}{*}{$18: 2 n-6$} & $20: 2 n-6$ & $1.2 \pm 0.0$ & $14.8 \pm 0.9$ & $5.8 \pm 0.2$ & $\mathrm{C} 18 \rightarrow 20$ \\
\hline & $22: 2 n-6$ & $1.4 \pm 0.0$ & $1.8 \pm 0.3$ & $1.5 \pm 0.0$ & $\mathrm{C} 20 \rightarrow 22$ \\
\hline & $24: 2 n-6$ & ND & ND & ND & $\mathrm{C} 22 \rightarrow 24$ \\
\hline & Total & $2.6 \pm 0.0$ & $16.5 \pm 1.0$ & $7.3 \pm 0.3$ & \\
\hline \multirow[t]{4}{*}{$18: 4 n-3$} & $20: 4 n-3$ & $1.4 \pm 0.3$ & $5.5 \pm 0.3$ & $4.0 \pm 0.2$ & $\mathrm{C} 18 \rightarrow 20$ \\
\hline & $22: 4 n-3$ & ND & $0.6 \pm 0.1$ & $0.5 \pm 0.0$ & $\mathrm{C} 20 \rightarrow 22$ \\
\hline & $24: 4 n-3$ & ND & ND & ND & $\mathrm{C} 22 \rightarrow 24$ \\
\hline & Total & $1.4 \pm 0.3$ & $6.0 \pm 0.3$ & $4.5 \pm 0.2$ & \\
\hline \multirow[t]{3}{*}{$18: 3 n-6$} & $20: 3 n-6$ & ND & $7.2 \pm 0.7$ & $5.0 \pm 0.1$ & $\mathrm{C} 18 \rightarrow 20$ \\
\hline & $22: 3 n-6$ & ND & $1.2 \pm 0.4$ & $0.7 \pm 0.1$ & $\mathrm{C} 20 \rightarrow 22$ \\
\hline & $24: 3 n-6$ & ND & ND & ND & $\mathrm{C} 22 \rightarrow 24$ \\
\hline
\end{tabular}

Results are expressed as the percentage of the fatty acid substrate converted to elongated fatty acid products with the following calculation: [individual product area / (all products area + substrate area)] $\times 100$. Value is presented as the mean $\pm \operatorname{SEM}(n=3)$.

Total conversion is calculated based on summation of all of the elongated products.

ND = not detected; SFA= Saturated fatty acid; PUFA= Polyunsaturated fatty acid 


\begin{tabular}{|c|c|c|c|c|c|}
\hline \multirow[t]{2}{*}{ Substrate } & \multirow{2}{*}{$\begin{array}{l}\text { Product } \\
\text { Total }\end{array}$} & \multicolumn{3}{|c|}{ Conversion rate (\%) } & \multirow[t]{2}{*}{ Activity } \\
\hline & & & $8.3 \pm 0.3$ & $5.7 \pm 0.2$ & \\
\hline \multirow[t]{7}{*}{$20: 5 n-3$} & $22: 5 n-3$ & ND & $3.9 \pm 0.2$ & $5.7 \pm 0.8$ & $\mathrm{C} 20 \rightarrow 22$ \\
\hline & $24: 5 n-3$ & ND & $0.5 \pm 0.1$ & $1.2 \pm 0.2$ & $\mathrm{C} 22 \rightarrow 24$ \\
\hline & $26: 5 n-3$ & ND & ND & $0.2 \pm 0.0$ & $\mathrm{C} 24 \rightarrow 26$ \\
\hline & $28: 5 n-3$ & ND & ND & $0.1 \pm 0.0$ & $\mathrm{C} 26 \rightarrow 28$ \\
\hline & $30: 5 n-3$ & ND & ND & $0.2 \pm 0.0$ & $\mathrm{C} 28 \rightarrow 30$ \\
\hline & $32: 5 n-3$ & ND & ND & $1.1 \pm 0.1$ & $\mathrm{C} 30 \rightarrow 32$ \\
\hline & Total & & $4.4 \pm 0.3$ & $8.3 \pm 1.1$ & \\
\hline \multirow[t]{7}{*}{$20: 4 n-6$} & $22: 4 n-6$ & ND & $4.4 \pm 0.2$ & $10.5 \pm 2.0$ & $\mathrm{C} 20 \rightarrow 22$ \\
\hline & $24: 4 n-6$ & ND & $0.7 \pm 0.1$ & $2.8 \pm 0.5$ & $\mathrm{C} 22 \rightarrow 24$ \\
\hline & $26: 4 n-6$ & ND & $0.6 \pm 0.1$ & $0.6 \pm 0.1$ & $\mathrm{C} 24 \rightarrow 26$ \\
\hline & $28: 4 n-6$ & ND & ND & $0.3 \pm 0.1$ & $\mathrm{C} 26 \rightarrow 28$ \\
\hline & $30: 4 n-6$ & ND & ND & $2.4 \pm 0.3$ & $\mathrm{C} 28 \rightarrow 30$ \\
\hline & $32: 4 n-6$ & ND & ND & $3.8 \pm 0.5$ & $\mathrm{C} 30 \rightarrow 32$ \\
\hline & Total & & $5.1 \pm 0.3$ & $20.4 \pm 2.8$ & \\
\hline \multirow[t]{6}{*}{$22: 5 n-3$} & $24: 5 n-3$ & ND & $1.5 \pm 0.1$ & $3.1 \pm 0.1$ & $\mathrm{C} 22 \rightarrow 24$ \\
\hline & $26: 5 n-3$ & ND & ND & $0.2 \pm 0.1$ & $\mathrm{C} 24 \rightarrow 26$ \\
\hline & $28: 5 n-3$ & ND & ND & $0.2 \pm 0.1$ & $\mathrm{C} 26 \rightarrow 28$ \\
\hline & $30: 5 n-3$ & ND & ND & $0.5 \pm 0.1$ & $\mathrm{C} 28 \rightarrow 30$ \\
\hline & $32: 5 n-3$ & ND & ND & $2.2 \pm 0.2$ & $\mathrm{C} 30 \rightarrow 32$ \\
\hline & Total & & $1.5 \pm 0.1$ & $6.1 \pm 0.2$ & \\
\hline \multirow[t]{3}{*}{$22: 4 n-6$} & $24: 4 n-6$ & ND & $1.7 \pm 0.2$ & $2.4 \pm 0.4$ & $\mathrm{C} 22 \rightarrow 24$ \\
\hline & $26: 4 n-6$ & ND & ND & $0.3 \pm 0.1$ & $\mathrm{C} 24 \rightarrow 26$ \\
\hline & $28: 4 n-6$ & ND & ND & $0.2 \pm 0.0$ & $\mathrm{C} 26 \rightarrow 28$ \\
\hline
\end{tabular}

Results are expressed as the percentage of the fatty acid substrate converted to elongated fatty acid products with the following calculation: [individual product area / (all products area + substrate area)] $\times 100$. Value is presented as the mean $\pm \operatorname{SEM}(n=3)$.

Total conversion is calculated based on summation of all of the elongated products.

ND = not detected; SFA= Saturated fatty acid; PUFA= Polyunsaturated fatty acid 


\begin{tabular}{|c|c|c|c|c|c|}
\hline \multirow[t]{2}{*}{ Substrate } & \multirow{2}{*}{$\begin{array}{l}\text { Product } \\
30: 4 n-6\end{array}$} & \multicolumn{3}{|c|}{ Conversion rate (\%) } & \multirow{2}{*}{$\begin{array}{l}\text { Activity } \\
\text { C28 } \rightarrow 30\end{array}$} \\
\hline & & ND & ND & $1.6 \pm 0.3$ & \\
\hline & $32: 4 n-6$ & ND & ND & $2.6 \pm 0.5$ & $\mathrm{C} 30 \rightarrow 32$ \\
\hline & Total & & $1.7 \pm 0.2$ & $7.1 \pm 1.1$ & \\
\hline $22: 6 n-3$ & $24: 6 n-3$ & ND & ND & ND & $\mathrm{C} 22 \rightarrow 24$ \\
\hline \multicolumn{6}{|c|}{$\begin{array}{l}\text { Results are expressed as the percentage of the fatty acid substrate converted to elongated fatty acid } \\
\text { products with the following calculation: [individual product area / (all products area + substrate area } \\
\times 100 \text {. Value is presented as the mean } \pm \text { SEM }(n=3) \text {. }\end{array}$} \\
\hline \multicolumn{6}{|c|}{ Total conversion is calculated based on summation of all of the elongated products. } \\
\hline $\mathrm{ND}=\operatorname{not} \mathrm{d}$ & $d ; S F A=S$ & ted & Polyuns & fatty acid & \\
\hline
\end{tabular}

Fatty acid composition of $P$. micromegethes

The FA composition of $P$. micromegethes whole body showed a higher proportion of SFAs (Table 6). Among these, $\mathrm{C} 16: 0$ is the predominant FA, while for monounsaturated fatty acids (MUFAs), C18:1n-7 was the most abundance. As for PUFA, the proportion of the total $n-6$ PUFA is twice higher than the n-3 PUFA, with significant contribution from LA and ARA. Within the n-3 PUFA, the percentage of DHA is also higher than EPA. 
Table 6

Whole body fatty acid composition of $P$. micromegethes

\section{Fatty acid}

C14:0

C15:0

C16:0

C17:0

C18:0

$\sum$ SFAs

C14:1

C15:1

C16:1

C17:1

C18:1n-9

C18:1n-7

C20:1n-9

$\sum$ MUFAs

C18:3n-3 (ALA)

C18:4n-3

C20:3n-3

C20:4n-3

C20:5n-3 (EPA)

C22:5n-3

C22:6n-3 (DHA)

C18:2n-6 (LA)

C18:3n-6

C20:3n-6

C20:4n-6 (ARA)

\section{Total fatty acid detected (\%)}

$2.0 \pm 0.1$

$1.6 \pm 0.2$

$29.8 \pm 1.3$

$3.0 \pm 0.4$

$16.9 \pm 0.6$

$53.4 \pm 1.7$

$1.3 \pm 0.1$

$0.7 \pm 0.1$

$3.8 \pm 0.4$

$0.5 \pm 0.0$

$0.9 \pm 0.3$

$15.8 \pm 0.3$

$0.4 \pm 0.0$

$23.4 \pm 0.5$

$0.4 \pm 0.1$

$0.2 \pm 0.0$

$-$

$0.2 \pm 0.0$

$0.3 \pm 0.0$

$0.3 \pm 0.0$

$4.7 \pm 0.8$

$8.0 \pm 0.6$

$0.4 \pm 0.0$

$0.5 \pm 0.1$

$6.3 \pm 0.7$

SFA = Saturated fatty acid; MUFA = Monounsaturated fatty acid; PUFA = Polyunsaturated fatty acid; $\mathrm{ALA}=\mathrm{a}$-Linolenic acid; $\mathrm{EPA}=$ Eicosapentaenoic acid; $\mathrm{DHA}=$ Docosahexaenoic acid; $\mathrm{LA}=$ Linoleic acid; $A R A=$ Arachidonic acid. Values are presented as the Mean \pm SEM of triplicate measurement. 


\begin{tabular}{|ll|}
\hline Fatty acid & Total fatty acid detected (\%) \\
\hline$\sum$ PUFAs & $21.4 \pm 2.0$ \\
\hline$\sum \mathrm{n}-3$ & $6.2 \pm 0.8$ \\
\hline$\sum \mathrm{n}-6$ & $15.2 \pm 1.2$ \\
$\mathrm{n}-3 / \mathrm{n}-6$ & $0.4 \pm 0.0$ \\
\hline $\begin{array}{l}\text { SFA = Saturated fatty acid; MUFA = Monounsaturated fatty acid; PUFA = Polyunsaturated fatty acid; } \\
\text { ALA = a-Linolenic acid; EPA = Eicosapentaenoic acid; DHA = Docosahexaenoic acid; LA = Linoleic } \\
\text { acid; ARA = Arachidonic acid. Values are presented as the Mean } \pm \text { SEM of triplicate measurement. }\end{array}$ \\
\hline
\end{tabular}

\section{Discussion}

Studies in different fish species have shown diverse combinations of Fads and Elovl orthologs, coupled with differences in substrate specificity and activity rate for both enzymes (Xie et al. 2021). The repertoire of LC-PUFA biosynthesizing Fads and Elovl in teleost was initially linked to species habitat, with species living in the LC-PUFA rich marine habitat displaying a loss of biosynthesis activities, presumably due the lack of evolutionary pressure to retain the biosynthesis pathway (Tocher et al. 2006; Zheng et al. 2009). The presence of multiple Fad and Elovl isoforms in Atlantic salmon was also linked to the transition from marine to freshwater lifestyle in this species (Monroig et al. 2010b; Morais et al. 2009; Zheng et al. 2005). Subsequently, the LC-PUFA biosynthesis capacity was hypothesized to be associated with the species trophic level (Li et al. 2010; Morais et al. 2012) and taxonomy position (Garrido et al. 2019).

The miniaturized cyprinid $P$. micromegethes, with its reduced smaller genome size and adaptation to peat swamp habitat offer insights into the relationship between an extreme habitat niche and biosynthesis of LC-PUFA (Malmstrøm et al. 2018). The use of RNA-seq data has become a cost-effective and useful approach for functional genomics research in species with or without a reference genome. De novo transcriptome assembly has been applied in a terrestrial crustacean species to mine for Fads or Elovl orthologs (Ting et al. 2021). Through the $P$. micromegethes transcriptome assembly, a single Fads 2 and four Elovl sequences were putatively identified. This cluster is consistent with the known orthologs in various Cyprinidae representatives (Agaba et al. 2004; Garrido et al. 2020; Hastings et al. 2001; Janaranjani et al. 2018; Janaranjani and Shu-Chien 2020; Monroig et al. 2010a; Sam et al. 2021).

The $P$. micromegethes Fads2 transcript encompassed all typical characteristics of a front-end desaturase. The presence of a cytochrome $b_{5}$-like domain at the $\mathrm{N}$-terminal end enables the electrontransfer process during the desaturation reaction and is obligatory for the catalytic activities of all frontend desaturases (Napier et al. 2003; Schenkman and Jansson 2003). Located within this domain is a HPGG motif, which forms the heme-binding core of the protein (Sayanova et al. 1999). Additionally, the sequence also consists of three conserved histidine boxes $(\mathrm{HxxxH}, \mathrm{HxxxHH}$ and QxxHH) pivotal for Fads catalytic activities (Hashimoto et al. 2008; Shanklin et al. 1994). Among front-end desaturases, the substitution of histidine ' $\mathrm{H}$ ' with glutamine 'Q' in the third histidine box QxxHH is essential for $\Delta 6$ 
desaturase activity and conserved among all teleost Fads2 (Castro et al. 2016; Sayanova et al. 2001). The four amino acid residues FQFQ situated between the second and third histidine boxes of the putative P. micromegethes Fads2 sequence has also been reported in other cyprinid fish (Garrido et al. 2020; Hastings et al. 2001; Janaranjani et al. 2018; Ren et al. 2013; Sam et al. 2021). This region was previously postulated to influence the Fads selectivity towards $\Delta 6 / \Delta 5$ or $\Delta 4$ substrates (Lim et al. 2014).

Phylogenetic tree built with Fads1 and Fads2 amino acid sequences from various orthologs placed the $P$. micromegethes Fads within the Fads2 clade, clustered within other Cypriniformes orthologs. This clade shares a similar ancestor with Fads2 from Siluriformes and Characiformes fish. Another larger Fads2 clade consist of orthologs from a diverse taxonomy group, including the ancient Ostoglossomorpha, Salmoniformes and Acanthomorpha. The Fads1 ortholog is found in Agnathans, Chondrichthyes, nonteleost ray-finned fish and early teleost groups such as Anguilla japonica. Several works have established the $\Delta 5$ function of these Fads 1 orthologs, which complement the $\Delta 6$ activity of the Fads2 (Castro et al. 2016; Lopes-Marques et al. 2018). After the 3R whole genome duplication (3R WGD), the loss of Fads1 occurred in most surviving teleost, which was subsequently compensated by the sub-functionalization of Fads2 to acquire the required $\Delta 5$ function (Lopes-Marques et al. 2017). Taken together, the sequence alignment and phylogenetic analysis suggest the isolation of a Fads2 from P. micromegethes.

All the four $P$. micromegethes Elovl sequences possess the characteristic features of an Elovl elongase, namely four conserved motifs (KXXEXXDT, QXXFLHXXHH, NXXXHXXMYXYY, and TXXQXX), seven transmembrane domains, a histidine rich box $(\mathrm{HXXHH})$ and a predicted ER retrieval signal with lysine $(\mathrm{K})$ or arginine (R) residues located near the carboxyl terminus (Jakobsson et al. 2006). The highly conserved $\mathrm{HXXHH}$ box is responsible for the electron transfer process during elongation (Leonard et al. 2004). Phylogenetic analysis of the four isolated Elovl transcripts placed these sequences to the respective Elovl5, Elovl2, Elovl4a, and Elovl4b clades. In early pre-teleost lineages such as lampreys and chondrichthyans, separate orthologues of Elovl5 and Elovl2 can be observed, as result of genome duplications in vertebrate ancestry (Monroig et al. 2016). Similarly, both these families are also present in early teleost groups, such as Anguilliformes and Otomorpha (Ferraz et al. 2019; Garrido et al. 2020; Janaranjani and Shu-Chien 2020; Machado et al. 2018; Oboh et al. 2016; Sam et al. 2021; Xu et al. 2020). The salmonids is the last teleost group retaining Elovl2 as this ortholog is missing in Acanthomorphans (Morais et al. 2009). Both the putative P. micromegethes Elovl4 sequences were placed in two separate clades comprising of the Elovl $4 \mathrm{a}$ and Elovl4b, respectively. The existence of two Elovl4 paralogues in modern teleost groups are speculated to be the consequence of teleost specific 3R WGD.

Functional characterization of the $P$. micromegethes Fads2 revealed a Fads2 with bifunctional $\Delta 6 / \Delta 5$ activities. This recapitulates the activities reported in zebrafish (Hastings et al. 2001), tench (Garrido et al. 2020), silver barb (Janaranjani et al. 2018) and mahseer (Sam et al. 2021). The $\Delta 6 / \Delta 5$ steps fulfil all the required desaturation steps for the biosynthesis of EPA and ARA. Additionally, the $\Delta 6$ desaturation of 24:5n-3 was also present, implying the utilization of the 'Sprecher' route for the conversion of EPA to DHA, which is typical of cyprinids. The $\Delta 5$ activity, while present in Fads2 of numerous freshwater teleosts and several marine species, are absent in most of the carnivorous marine teleost studied so far (Mohd-Yusof

Page 18/32 
et al. 2010; Morais et al. 2011; Zheng et al. 2009). This impairment could ostensibly be the result of the LC-PUFA rich prey these species consumed (Tocher et al. 2006). Additionally, there was no prominent $\Delta 8$ or $\Delta 4$ activities in the $P$. micromegethes Fads2. In mammals and teleost, the $\Delta 8$ step offers the possibility of an alternate direction for EPA synthesis besides the conventional $\Delta 6$ route (Monroig et al. 2011a; Park et al. 2009). While some bifunctional $\Delta 6 / \Delta 5$ Fads2 or $\Delta 6$ Fads2 were reported to have $\Delta 8$ activity, overall findings seemed to suggest marine teleost conspicuously utilizing the $\Delta 8$ pathway as compared to freshwater or diadromous fish (Monroig et al. 2011a). The presence of $\Delta 4$ Fads in the more recent teleost lineages suggest the $\Delta 4$ pathway to be comparatively recent in teleost evolution and hence, missing in earlier lineages such as cyprinids (Oboh et al. 2017a).

The P. micromegethes Elovl5 showed elongation capacity towards C18 and C20 PUFAs, without any activity towards $\mathrm{C} 22$ substrates. This preference towards C18-C20 PUFA substrates is similar with most of the Elovl5 orthologs (Monroig et al. 2016). Between the C18 substrates, P. micromegethes Elovl5 exhibited substantially higher activities towards C18:4n-3 or C18:3n-6, as compared to the ALA or LA. This befits the $\Delta 6$ desaturation of ALA or LA as the first step of the biosynthesis pathway, as compared to the $\Delta 8$ path (Monroig et al. 2011a). The lack of elongation activity towards C22 PUFA substrates is commonly reported in teleost Elovl5 orthologs, except for orthologs from several marine species (Monroig et al. 2012; Soo et al. 2020; Xie et al. 2016). The P. micromegethes Elovl2 appears to be an elongase with wide range of substrate specificity. More importantly, this Elovl2 could elongate C22 PUFA substrates to their respective C24 PUFA substrates. Since the first discovery of Elovl4 in zebrafish, subsequent findings have showed its capacity for VLC-PUFA elongation (Ferraz et al. 2020; Monroig et al. 2010a; Monroig et al. 2012; Monroig et al. 2011b; Oboh et al. 2017b). While freshwater fish species such as cyprinids could potentially utilize Elovl4 for C22 PUFA elongation, Elovl4 is postulated to compensate for the loss of Elovl2 in marine species (Betancor et al. 2020; Luo et al. 2021). Between the two isoforms, the $P$. micromegethes Elovl4a displayed higher elongation activities towards C18 PUFA while the Elovl4b showed higher activities towards the $\mathrm{C} 20$ substrates. Majority of the studied teleost species also reported higher rate of C20 and C22 PUFA elongation with Elovl4b (Ferraz et al. 2020; Monroig et al. 2010a; Morais et al. 2020). In contrast, the African catfish Elovl4a showed higher activities among the two paralogs (Oboh et al. 2017b). The elongation of $\mathrm{C} 24: 0$ by P. micromegethes Elovl4 is consistent with SFA elongation activities observed in both freshwater and marine species (Ferraz et al. 2020; Janaranjani and Shu-Chien 2020; Morais et al. 2020). It is worth mentioning that a new Elovl8 family, was recently described in teleost, and was shown to play a role in elongation of PUFA substrates (Li et al. 2020; Sun et al. 2021). Although hits matching Elovl 8 were found in $P$. micromegethes, these sequences were not further studied as all the steps required for LC-PUFA biosynthesis are already fulfilled by the above described Elovl families.

Taken together, the functional characterization of $P$. micromegethes Fads, Elov15, Elovl2, and Elovl4 showed that $P$. micromegethes possess a complete LC-PUFA biosynthesis pathway. The detection of intermediates PUFA such as C18:4n-3, C20:4n-3 and C22:5n-3 in P. micromegethes whole body also suggest biosynthesis activities in vivo. Like other cyprinids, $P$. micromegethes whole body also contain higher proportion of DHA than EPA (Jaya-Ram et al. 2018). While the biosynthesis of EPA and ARA 
follows the conventional $\Delta 6$ route, the conversion of EPA to DHA utilizes the 'Sprecher' route (Fig. 5). This route is conserved in all studied otomorphans, except for a marine clupeid species which possess a Fads2 devoid of $\Delta 5$ capacity (Ferraz et al. 2019; Garrido et al. 2020; Janaranjani et al. 2018; Janaranjani and Shu-Chien 2020; Machado et al. 2018; Oboh et al. 2016; Sam et al. 2021). This finding supports the suggestion that the phylogeny position of a fish species takes preeminence over trophic level as a factor influencing the LC-PUFA biosynthesis capacity of a species (Galindo et al. 2021; Garrido et al. 2019). Beside these species, the Atlantic salmon and rainbow trout also utilized a similar route for the biosynthesis of EPA and DHA (Abdul Hamid et al. 2016; Hastings et al. 2004; Monroig et al. 2010b; Oboh et al. 2017a). Therefore, despite having a relatively reduced genome size as compared to other cyprinids, the LC-PUFA biosynthesis capacity in P. micromegethes remain intact (Malmstrøm et al. 2018). The retaining of this pathway indicates the importance of LC-PUFA biosynthesis for life in peat swamps. Due to the extreme acidic condition and poor light penetration, peat swamp water bodies are characterized by low plankton abundance and limited availability of nutrients (Helmizuryani et al. 2020; Yule and Gomez 2009).

\section{Conclusions}

A Fads2 and Elovl5, Elovl2 and Elovl4 orthologs were mined from a high quality de novo transcriptome assembly of a miniaturized cyprinid, P. micromegethes. Using yeast-based heterologous expression approach, the functional characterization of each orthologs were studied. Collectively, results showed that $P$. micromegethes possess all the necessary Fads and Elovl for the biosynthesis of LC-PUFA from C18 PUFA substrates.

\section{Declarations}

Acknowledgements We would like to thank to Michael L.S.H. for his support during the fieldwork.

\section{Statements and Declarations}

Funding This work was supported by the Vice-Chancellor of Universiti Sains Malaysia, USMStrategic Initiative-Ten Q1Q2, Grant number: 311. PCCB.411954.

Conflict of interest The authors declare that they have no conflict of interest.

Author contributions Conceptualization and funding: AC Shu-Chien. Planning of experiments: AC Shu-Chien, Nyok-Sean L, Meng-Kiat K and Ka-Kei S. Experiment: Ka-Kei S and Nyok-Sean L. Analysis: AC Shu-Chien, Nyok-Sean L and Ka-Kei S. Writing and editing of manuscript: AC Shu-Chien, Nyok-Sean L, Meng-Kiat K, Ka-Kei S, Lading E.

Data Availability This current research was registered in NCBI database under BioProjet ID PRJNA737281. The datasets generated during and/or analyzed during this current research were deposited in the NCBI Sequence Read Archive (SRA) under accession number SRP323968. 
Code availability Not applicable.

Ethics approval This study was performed in accordance with the USM Institutional Animal Care and Use Committee guidelines under the approval code USM/IACUC/2019/(117)(988).

Consent to participate Not applicable.

Consent for publication All the authors have approved the final manuscript.

\section{References}

1. Abdul Hamid NK, Carmona-Antonanzas G, Monroig O, Tocher DR, Turchini GM, Donald JA (2016) Isolation and functional characterisation of a fads2 in rainbow trout (Oncorhynchus mykiss) with $\Delta 5$ desaturase activity. PLoS ONE 11(3):e0150770. doi:10.1371/journal.pone.0150770

2. Agaba M, Tocher DR, Dickson CA, Dick JR, Teale AJ (2004) Zebrafish cDNA encoding multifunctional fatty acid elongase involved in production of eicosapentaenoic (20:5n-3) and docosahexaenoic (22:6n-3) acids. Mar Biotechnol 6(3):251-261. doi:10.1007/s10126-003-0029-1

3. Berra TM (2001) Freshwater Fish Distribution. Academic Press, San Diego

4. Betancor MB et al (2020) Molecular and functional characterisation of a putative elovl4 gene and its expression in response to dietary fatty acid profile in Atlantic bluefin tuna (Thunnus thynnus). Comp Biochem Physiol B Biochem Mol Biol 240:110372. doi:10.1016/j.cbpb.2019.110372

5. Britz R, Kottelat M (2008) Paedocypris carbunculus, a new species of miniature fish from Borneo (Teleostei: Cypriniformes: Cyprinidae). The Raffles Bulletin of Zoology 56:415-422

6. Castro LFC, Monroig Ó, Leaver MJ, Wilson J, Cunha I, Tocher DR (2012) Functional desaturase Fads1 $\Delta 5$ and Fads $2 \Delta 6$ orthologues evolved before the origin of jawed vertebrates. PLoS ONE 7(2):e31950. doi:10.1371/journal.pone.0031950

7. Castro LFC, Tocher DR, Monroig O (2016) Long-chain polyunsaturated fatty acid biosynthesis in chordates: Insights into the evolution of Fads and Elovl gene repertoire. Prog Lipid Res 62:25-40. doi:10.1016/j.plipres.2016.01.001

8. Conesa A, Götz S, García-Gómez JM, Terol J, Talón M, Robles M (2005) Blast2GO: a universal tool for annotation, visualization and analysis in functional genomics research. Bioinformatics 21(18):3674-3676. doi:10.1093/bioinformatics/bti610

9. Ferraz RB et al (2019) A complete enzymatic capacity for long-chain polyunsaturated fatty acid biosynthesis is present in the Amazonian teleost tambaqui, Colossoma macropomum. Comp Biochem Phys B 227:90-97. doi:https://doi.org/10.1016/j.cbpb.2018.09.003

10. Ferraz RB et al (2020) The fatty acid elongation genes elovl4a and elovl4b are present and functional in the genome of tambaqui (Colossoma macropomum). Comp Biochem Physiol B 245:110447. doi:https://doi.org/10.1016/j.cbpb.2020.110447 
11. Galindo A et al (2021) Polyunsaturated fatty acid metabolism in three fish species with different trophic level. Aquaculture 530:735761. doi:https://doi.org/10.1016/j.aquaculture.2020.735761

12. Garrido $D$ et al (2019) Functional diversification of teleost Fads2 fatty acyl desaturases occurs independently of the trophic level. Sci Rep 9(1):11199. doi:10.1038/s41598-019-47709-0

13. Garrido D et al (2020) Lipid metabolism in Tinca tinca and its n-3 LC-PUFA biosynthesis capacity. Aquaculture 523:735147. doi:https://doi.org/10.1016/j.aquaculture.2020.735147

14. Gladyshev MI, Arts MT, Sushchik NN (2009) Preliminary estimates of the export of omega-3 highly unsaturated fatty acids (EPA+DHA) from aquatic to terrestrial ecosystems. In: Kainz M, Brett MT, Arts MT (eds) Lipids in Aquatic Ecosystems. Springer New York, New York, NY, pp 179-210

15. Grabherr MG et al (2011) Full-length transcriptome assembly from RNA-Seq data without a reference genome. Nat Biotechnol 29(7):644-652. doi:10.1038/nbt.1883

16. Hashimoto K, Yoshizawa AC, Okuda S, Kuma K, Goto S, Kanehisa M (2008) The repertoire of desaturases and elongases reveals fatty acid variations in 56 eukaryotic genomes. J Lipid Res 49(1):183-191. doi:10.1194/jlr.M700377-JLR200

17. Hastings $\mathrm{N}$ et al (2001) A vertebrate fatty acid desaturase with $\Delta 5$ and $\Delta 6$ activities. Proc Natl Acad Sci USA 98(25):14304-14309. doi:10.1073/pnas.251516598

18. Hastings $\mathrm{N}$ et al (2004) Molecular cloning and functional characterization of fatty acyl desaturase and elongase cDNAs involved in the production of eicosapentaenoic and docosahexaenoic acids from a-linolenic acid in Atlantic Salmon (Salmo salar). Mar Biotechnol 6(5):463-474

19. Helmizuryani, Suwignyo RA, Hanafiahand Z, Faizal M (2020) The abundance and diversity of plankton on peat swamps area Ogan Komering Ilir (OKI) Regency, South Sumatera. IOP Conference Series: Earth and Environmental Science 584(1):012046 doi:10.1088/1755-1315/584/1/012046

20. Jakobsson A, Westerberg R, Jacobsson A (2006) Fatty acid elongases in mammals: Their regulation and roles in metabolism. Prog Lipid Res 45(3):237-249.

doi:http://dx.doi.org/10.1016/j.plipres.2006.01.004

21. Janaranjani $\mathrm{M}$ et al (2018) Capacity for eicosapentaenoic acid and arachidonic acid biosynthesis in silver barb (Barbonymus gonionotus): Functional characterisation of a $\Delta 6 / \Delta 8 / \Delta 5$ Fads2 desaturase and Elovl5 elongase. Aquaculture 497:469-486.

doi:https://doi.org/10.1016/j.aquaculture.2018.08.019

22. Janaranjani M, Shu-Chien AC (2020) Complete repertoire of long-chain polyunsaturated fatty acids biosynthesis enzymes in a cyprinid, silver barb (Barbonymus gonionotus): Cloning, functional characterization and dietary regulation of Elovl2 and Elovl4. Aquac Nutr 26(5):1835-1853. doi:https://doi.org/10.1111/anu.13133

23. Jaya-Ram A, Fuad F, Zakeyuddin MS, Sah ASRM (2018) Muscle Fatty Acid Content in Selected Freshwater Fish from Bukit Merah Reservoir, Perak, Malaysia. Tropical Life Science Research 29(2):103-117

24. Jump DB (2002) The biochemistry of n-3 polyunsaturated fatty acids. J Biol Chem 277(11):87558758. doi:10.1074/jbc.R100062200 
25. Kabeya $\mathrm{N}$ et al (2018) Genes for de novo biosynthesis of omega-3 polyunsaturated fatty acids are widespread in animals. Sci Adv 4(5):eaar6849-eaar6849. doi:10.1126/sciadv.aar6849

26. Kottelat M, Britz R, Hui TH, Witte K-E (2006) Paedocypris, a new genus of Southeast Asian cyprinid fish with a remarkable sexual dimorphism, comprises the world's smallest vertebrate. Proc Biol Sci 273(1589):895-899

27. Kriventseva EV, Zdobnov EM, Simão FA, loannidis P, Waterhouse RM (2015) BUSCO: assessing genome assembly and annotation completeness with single-copy orthologs. Bioinformatics (Oxford England) 31(19):3210-3212. doi:10.1093/bioinformatics/btv351

28. Kuah M-K, Jaya-Ram A, Shu-Chien AC (2015) The capacity for long-chain polyunsaturated fatty acid synthesis in a carnivorous vertebrate: Functional characterisation and nutritional regulation of a Fads2 fatty acyl desaturase with $\Delta 4$ activity and an Elovl5 elongase in striped snakehead (Channa striata). BBA-Mol Cell Biol Lipids 1851(3):248-260. doi:http://dx.doi.org/10.1016/j.bbalip.2014.12.012

29. Kuah M-K, Jaya-Ram A, Shu-Chien AC (2016) A fatty acyl desaturase (fads2) with dual $\Delta 6$ and $\Delta 5$ activities from the freshwater carnivorous striped snakehead Channa striata. Comp Biochem Physiol A: Mol Integr Physiol 201:146-155. doi:https://doi.org/10.1016/j.cbpa.2016.07.007

30. Leonard AE, Pereira SL, Sprecher H, Huang Y-S (2004) Elongation of long-chain fatty acids. Prog Lipid Res 43(1):36-54. doi:http://dx.doi.org/10.1016/S0163-7827(03)00040-7

31. Li W, Godzik A (2006) Cd-hit: a fast program for clustering and comparing large sets of protein or nucleotide sequences. Bioinformatics (Oxford England) 22(13):1658-1659. doi:10.1093/bioinformatics/btl158

32. Li Y et al (2010) Vertebrate fatty acyl desaturase with $\Delta 4$ activity. Proc Natl Acad Sci USA 107(39):16840-16845. doi:10.1073/pnas.1008429107

33. Li Y et al (2020) Genome wide identification and functional characterization of two LC-PUFA biosynthesis elongase (elovl8) genes in rabbitfish (Siganus canaliculatus). Aquaculture 522:735127. doi:https://doi.org/10.1016/j.aquaculture.2020.735127

34. Lim Z, Senger T, Vrinten $P$ (2014) Four amino acid residues influence the substrate chain-length and regioselectivity of Siganus canaliculatus $\Delta 4$ and $\Delta 5 / 6$ desaturases. Lipids 49(4):357-367. doi:10.1007/s11745-014-3880-0

35. Lopes-Marques M et al (2018) Retention of fatty acyl desaturase 1 (fads 1 ) in Elopomorpha and Cyclostomata provides novel insights into the evolution of long-chain polyunsaturated fatty acid biosynthesis in vertebrates. BMC Evol Biol 18(1):157. doi:10.1186/s12862-018-1271-5

36. Lopes-Marques M, Ozorio R, Amaral R, Tocher DR, Monroig O, Castro LFC (2017) Molecular and functional characterization of a fads2 orthologue in the Amazonian teleost, Arapaima gigas. Comp Biochem Phys B 203:84-91. doi:10.1016/j.cbpb.2016.09.007

37. Luo J et al (2021) Biosynthesis of LC-PUFAs and VLC-PUFAs in Pampus argenteus: Characterization of Elovl4 Elongases and Regulation under Acute Salinity. J Agric Food Chem 69(3):932-944. doi:10.1021/acs.jafc.0c06277 
38. Machado AM et al (2018) "Out of the Can": A Draft Genome Assembly, Liver Transcriptome, and Nutrigenomics of the European Sardine, Sardina pilchardus. Genes 9(10):485

39. Malmstrøm M et al (2018) The most developmentally truncated fishes show extensive Hox gene loss and miniaturized genomes. Genome Biology and Evolution:

evy058-evy058

. doi:10.1093/gbe/evy058

40. Matsushita $Y$ et al (2020) Flatfishes colonised freshwater environments by acquisition of various DHA biosynthetic pathways. Commun Biol 3(1):516. doi:10.1038/s42003-020-01242-3

41. Mohd-Yusof NY, Monroig O, Mohd-Adnan A, Wan KL, Tocher DR (2010) Investigation of highly unsaturated fatty acid metabolism in the Asian sea bass, Lates calcarifer. Fish Physiol Biochem 36(4):827-843. doi:10.1007/s10695-010-9409-4

42. Monroig O, Li Y, Tocher DR (2011a) Delta-8 desaturation activity varies among fatty acyl desaturases of teleost fish: High activity in delta-6 desaturases of marine species. Comp Biochem Phys B 159(4):206-213. doi:10.1016/j.cbpb.2011.04.007

43. Monroig Ó et al (2016) Evolutionary functional elaboration of the Elovl2/5 gene family in chordates. Sci Rep 6:20510. doi:10.1038/srep20510

44. Monroig O, Rotllant J, M C-RJ, Dick JR, Figueras A, Tocher DR (2010a) Expression and role of Elovl4 elongases in biosynthesis of very long-chain fatty acids during zebrafish Danio rerio early embryonic development. BBA-Mol Cell Biol Lipids 1801:1145-1154

45. Monroig Ó, Wang S, Zhang L, You C, Tocher DR, Li Y (2012) Elongation of long-chain fatty acids in rabbitfish Siganus canaliculatus: Cloning, functional characterisation and tissue distribution of Elovl5- and Elovl4-like elongases. Aquaculture 350-353(0):63-70. doi:http://dx.doi.org/10.1016/j.aquaculture.2012.04.017

46. Monroig Ó, Webb K, Ibarra-Castro L, Holt GJ, Tocher DR (2011b) Biosynthesis of long-chain polyunsaturated fatty acids in marine fish: Characterization of an Elovl4-like elongase from cobia Rachycentron canadum and activation of the pathway during early life stages. Aquaculture 312(1):145-153. doi:https://doi.org/10.1016/j.aquaculture.2010.12.024

47. Monroig O, Zheng X, Morais S, Leaver MJ, Taggart JB, Tocher DR (2010b) Multiple genes for functional 6 fatty acyl desaturases (Fad) in Atlantic salmon (Salmo salarL.): gene and cDNA characterization, functional expression, tissue distribution and nutritional regulation. Biochim Biophys Acta 1801(9):1072-1081. doi:10.1016/j.bbalip.2010.04.007

48. Morais S, Castanheira F, Martinez-Rubio L, Conceição LEC, Tocher DR (2012) Long chain polyunsaturated fatty acid synthesis in a marine vertebrate: Ontogenetic and nutritional regulation of a fatty acyl desaturase with $\Delta 4$ activity. BBA-Mol Cell Biol Lipids 1821(4):660-671. doi:https://doi.org/10.1016/j.bbalip.2011.12.011

49. Morais S, Monroig O, Zheng X, Leaver M, Tocher D (2009) Highly unsaturated fatty acid synthesis in Atlantic Salmon: characterization of ELOVL5- and ELOVL2-like elongases. Mar Biotechnol 11(5):627-639 
50. Morais S, Mourente G, Ortega A, Tocher JA, Tocher DR (2011) Expression of fatty acyl desaturase and elongase genes, and evolution of DHA:EPA ratio during development of unfed larvae of Atlantic bluefin tuna (Thunnus thynnus L.). Aquaculture 313(1):129-139.

doi:https://doi.org/10.1016/j.aquaculture.2011.01.031

51. Morais $S$ et al (2020) Molecular and Functional Characterization of Elovl4 Genes in Sparus aurata and Solea senegalensis Pointing to a Critical Role in Very Long-Chain ( $>\mathrm{C} 24)$ Fatty Acid Synthesis during Early Neural Development of Fish. Int J Mol Sci 21(10):3514

52. Napier JA, Michaelson LV, Sayanova O (2003) The role of cytochrome b5 fusion desaturases in the synthesis of polyunsaturated fatty acids. Prostaglandins Leukot Essent Fatty Acids 68(2):135-143. doi:https://doi.org/10.1016/S0952-3278(02)00263-6

53. Nguyen L-T, Schmidt HA, von Haeseler A, Minh BQ (2014) IQ-TREE: A Fast and Effective Stochastic Algorithm for Estimating Maximum-Likelihood Phylogenies. Mol Biol Evol 32(1):268-274. doi:10.1093/molbev/msu300

54. Oboh A, Betancor MB, Tocher DR, Monroig O (2016) Biosynthesis of long-chain polyunsaturated fatty acids in the African catfish Clarias gariepinus: Molecular cloning and functional characterisation of fatty acyl desaturase (fads2) and elongase (elovl2) cDNAs7. Aquaculture 462:70-79 doi:https://doi.org/10.1016/j.aquaculture.2016.05.018

55. Oboh A et al (2017a) Two alternative pathways for docosahexaenoic acid (DHA, 22:6n-3) biosynthesis are widespread among teleost fish. Sci Rep 7(1):3889. doi:10.1038/s41598-017-042882

56. Oboh A, Navarro-Guillén C, Tocher DR, Monroig O (2017b) Elongation of very long-chain (>C24) fatty acids in Clarias gariepinus: Cloning, functional characterization and tissue expression of elov/4 elongases. Lipids 52:837-848. doi:10.1007/s11745-017-4289-3

57. Park WJ, Kothapalli KSD, Lawrence P, Tyburczy C, Brenna JT (2009) An alternate pathway to longchain polyunsaturates: the FADS2 gene product $\triangle 8$-desaturates 20:2n-6 and 20:3n-3. J Lipid Res 50(6):1195-1202. doi:10.1194/jIr.M800630-JLR200

58. Ren HT et al (2013) Two $\Delta 6$-desaturase-like genes in common carp (Cyprinus carpio var. Jian): structure characterization, mRNA expression, temperature and nutritional regulation. Gene 525(1):11-17. doi:10.1016/j.gene.2013.04.073

59. Ruber L, Kottelat M, Tan HH, Ng PK, Britz R (2007) Evolution of miniaturization and the phylogenetic position of Paedocypris, comprising the world's smallest vertebrate. BMC Evol Biol 7:38. doi:10.1186/1471-2148-7-38

60. Sam K-K, Merosha P, Janaranjani M, Athirah I, Shu-Chien AC (2021) The Malaysian Mahseer, Tor tambroides possess all required biosynthesis enzymes for the conversion of $\mathrm{C} 18$ polyunsaturated fatty acids to long-chain polyunsaturated fatty acids. Aquaculture 543:736942. doi:https://doi.org/10.1016/j.aquaculture.2021.736942

61. Sayanova O, Beaudoin F, Libisch B, Castel A, Shewry PR, Napier JA (2001) Mutagenesis and heterologous expression in yeast of a plant $\Delta 6$-fatty acid desaturase. J Exp Bot 52(360):1581-1585. 
doi:10.1093/jexbot/52.360.1581

62. Sayanova O, Shewry PR, Napier JA (1999) Histidine-41 of the cytochrome b5 domain of the borage $\Delta 6$ fatty acid desaturase is essential for enzyme activity. Plant Physiol 121(2):641-646. doi:10.1104/pp.121.2.641

63. Schenkman JB, Jansson I (2003) The many roles of cytochrome b5. Pharmacol Ther 97(2):139152. doi:10.1016/s0163-7258(02)00327-3

64. Shanklin J, Whittle E, Fox BG (1994) Eight histidine residues are catalytically essential in a membrane-associated iron enzyme, stearoyl-CoA desaturase, and are conserved in alkane hydroxylase and xylene monooxygenase. Biochemistry 33(43):12787-12794. doi:10.1021/bi00209a009

65. Soo HJ et al (2020) Functional characterisation of fatty acyl desaturase, Fads2, and elongase, Elovl5, in the Boddart's goggle-eyed goby Boleophthalmus boddarti (Gobiidae) suggests an incapacity for long-chain polyunsaturated fatty acid biosynthesis. J Fish Biol 97(1):83-99. doi:10.1111/jfb.14328

66. Sprecher H (1981) Biochemistry of essential fatty acids. Prog Lipid Res 20:13-22

67. Sprecher H, Luthria DL, Mohammed BS, Baykousheva SP (1995) Reevaluation of the pathways for the biosynthesis of polyunsaturated fatty acids. J Lipid Res 36(12):2471-2477

68. Sun S et al (2021) Evolution and Functional Characteristics of the Novel elovl8 That Play Pivotal Roles in Fatty Acid Biosynthesis. Genes (Basel) 12(8). doi:10.3390/genes12081287

69. Ting SY et al (2021) Long-Read Sequencing Reveals the Repertoire of Long-Chain Polyunsaturated Fatty Acid Biosynthetic Genes in the Purple Land Crab, Gecarcoidea lalandii (H. Milne Edwards, 1837). Front Mar Sci 8(925). doi:10.3389/fmars.2021.713928

70. Tocher DR, Zheng X, Schlechtriem C, Hastings N, Dick J, Teale A (2006) Highly unsaturated fatty acid synthesis in marine fish: Cloning, functional characterization, and nutritional regulation of fatty acyl $\Delta 6$ desaturase of Atlantic cod (Gadus morhua L.). Lipids 41(11):1003-1016

71. Twining CW et al (2021) The evolutionary ecology of fatty-acid variation: Implications for consumer adaptation and diversification. Ecol Lett 24(8):1709-1731. doi:10.1111/ele.13771

72. Weitzman SH, Vari RP (1988) Miniaturization in South American freshwater fishes; an overview and discussion. Proceedings of the Biological Society of Washington 101:444-465

73. Xie D et al (2021) Regulation of long-chain polyunsaturated fatty acid biosynthesis in teleost fish. Prog Lipid Res 82:101095. doi:10.1016/j.plipres.2021.101095

74. Xie D et al (2016) Long-chain polyunsaturated fatty acid biosynthesis in the euryhaline herbivorous teleost Scatophagus argus: Functional characterization, tissue expression and nutritional regulation of two fatty acyl elongases. Comp Biochem Phys B 198:37-45. doi:http://dx.doi.org/10.1016/j.cbpb.2016.03.009

75. Xu W et al (2020) The catadromous teleost Anguilla japonica has a complete enzymatic repertoire for the biosynthesis of docosahexaenoic acid from a-linolenic acid: Cloning and functional 
characterization of an Elovl2 elongase. Comp Biochem Phys B 240:110373.

doi:https://doi.org/10.1016/j.cbpb.2019.110373

76. Yule CM, Gomez LN (2009) Leaf litter decomposition in a tropical peat swamp forest in Peninsular Malaysia. Wetlands Ecol Manage 17(3):231-241. doi:10.1007/s11273-008-9103-9

77. Zheng X, Ding Z, Xu Y, Monroig O, Morais S, Tocher DR (2009) Physiological roles of fatty acyl desaturases and elongases in marine fish: Characterisation of cDNAs of fatty acyl $\Delta 6$ desaturase and elovl5 elongase of cobia (Rachycentron canadum). Aquaculture 290(1-2):122-131

78. Zheng X, Tocher DR, Dickson CA, Bell JG, Teale AJ (2005) Highly unsaturated fatty acid synthesis in vertebrate: new insights with the cloning and characterization of a $\Delta 6$ desaturase of Atlantic salmon. Lipids 40(1):13-24

\section{Figures}


HsaFads2

Pmi Fads2

BgoFads2

DreFads2

CgaFads2

ScaFads2 $\Delta 4$

ScaFads $2-\Delta 6 / \Delta 5$

HsaFads2

Pmi Fads2

BgoFads2

DreFads2

CgaFads2

ScaFads $2 \Delta 4$

ScaFads $2-\Delta 6 / \Delta 5$

HsaFads2

Pmi Fads2

BgoFads2

DreFads2

CgaFads 2

ScaFads $2 \Delta 4$

ScaFads2 $\Delta 6 / \Delta 5$

HsaFads2

PmiFads2

BgoFads2

DreFads2

CgaFads 2

ScaFads $2 \Delta 4$

ScaFads2 $\Delta 6 / \Delta 5$

HsaFads2

Pmi Fads2

BgoFads2

DreFads2
HPGG

MGKGGNQGEG-AAEREVSVPT FSWEE IQKHNLRTDRWLVIDRKVYNITKWSIQHPGGQRV MGGGAQQTDR-I PGTSGRSGTYTWEEVQKHTKAGDQW IVVERKVYNVSQWVKRHPGGLR MGGGGQQTDR-IAGTNGRFSTYTWEEVKKHTKPEDOWIVVERKVYNVSOWVKRHPGGMRV MGGGGQQTDR-ITDTNGRESSYTWEEMQKHTKHGDQWVVVERKVYNVSQWVKRHPGGLRI MGGGGHRGEQLGSGETSSCKQYTWDEVQKHNRRGDQWVVIERKVYNVTEWTKRHPGGRRV MGGGGQLGESGENGCKSAAGVYTWEEVQHHSNRNDQWLVIDRKVYNVTQWAKRHPGGFRV

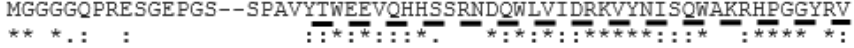

IGHYAGEDATDAFRAFHPDLE FVGKFLKPLLIGELAPEEPSQDHGKNSKITEDFRALRKT LGHYAGEDATEAFTAFHPDLQLVRKYLKPLMIGELEASE PSQDRQKNAALVEDFRALRER IRHYAGEDATDAFHAFHPNLOLVRKYMKPLLIGELEASE PSODGOKNGALVEDFRALREC LGHYAGEDATEAFTAFHPNLQLMRKYLKPLLIGELEASE PSQDRQKNAALVEDFRALRER LAHYAGEDATEAF IAFHPDQRFVRKYMKPLLVGELASSE PSQDHGKNVALVEDFETLRKQ LNHYAGEDATEAFTAFHPDIKFVQKYMKPLLVGELAATE PSQDQDKNAALIQDFHTLRQQ IGHYAGEDATEAFTAFHPDLKFVQKFLKPLLIGELAATE P SQDNKNAALIQDFHTLRQQ

AEDMNLFKTNHVFFLLLLAHI IALESIAWFTVFYFGNGW I PTLITAFVLATSQAQAGWLQ LEADGCFRTDPLF FLLHLGHI LLLEAVALMLVWYCGT GW ITTAVVAVLLATSQSQAGWLQ LEAEGCFKTOPLFFILHLGHILLLEAIALMMVWYFGTGNINTAIVAVILATAOSOAGWLO LEAEGCFKTQPLFFALHLGHILLLEAIAFVMVWYFGTGWINTLIVAVILATAQSQAGWLQ LEAQGLFRTSPLF F IYLGHILLLEGLSLALLWTFGNGWIITALISVILATAQAQAGWLQ AESEGLFQARPLF FLLHLGHI LLLEALALLMVWHWGT GWLQTLLCAVMLATAQSOAGWL AESEGLFQARPLFFLLHLGHILLLEALALLMVWHWGTGWLQTLLCAVMLATAQSQAGWLO

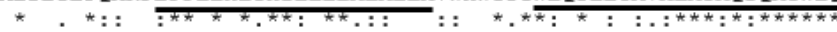
$\mathrm{HxxxH}$ $\mathrm{HxxHH}$

ADYGHLSVYRKPKWNHLVHKFVIGHLKGASANWWNHRHF QHHAKPNI FHKDPDVNMLHVF HDFGHLSVFKSSRWNHLVHKFVIGQLKGASAGWWNHRHFOHHAKPNVFKKDPDVNMLNAF MDGHLSVFKNSRWDHLLHKFVI GHLKGAAAGWWNHRHFOHHAKPNI FKKDPDINMISAF DFGHLSVFKT SGMNHLVHKFVI GHLKGASAGWWNHRHFQHHAKPNI FKKDPDVNMLNAF ADFGHLSVFKNSTWDHIMHKFVIGHLKGASANWWNHRHFQHHAKPNVISKDPDVNMINI DFGHLSVFKKSRWNHLVHKFVIGHLKGASANWWNHRHFQHHAKPNI FKKDPDINMVDL DFGHLSVFKKSRWNHLVHHFVIGHLKGASANWWNHRHF QHHAKPNI FKKDPDINMVDL

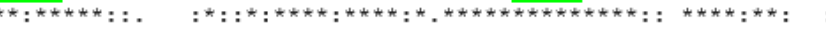

VLGEWQPIEYGKKKLKYLPYNHQHEYFFL I GP PLL I PMYFQYQI IMTMIVHKNWVDLAWA VVGKVQPVEYGVKKI KNLPYNHQHKYFFF I GPPLLI PVYFQFQI I HNMVAHGLWVDLVWC VVGKVOPVEYGIKKI KNLPYNHOHKYFFFIGPPLIL PVYFOFOI FHNMTMHGLWVDLAWC VVGNVQPVEYGVKKIKHLPYNHQHKYF FF I GPPLL I PVYFQFQ I FHNMISHGMWVDLLWC
179

179

179

179

180

239

239

239

239

240

240

238

299

299

299

299

\section{Figure 1}

Comparison of the deduced amino acid (aa) sequence of the cloned P. micromegethes Fads2 (UBB58395) and orthologues from Homo sapiens (AAD20018), Clarias gariepinus (AMR43366), Barbonymus gonionotus (AXF92413), Danio rerio (AAG25710), Siganus canaliculatus $\Delta 4$ (ADJ29913), and $S$. canaliculatus $\Delta 6 / \Delta 5$ (ABR12315) through alignment using Clustal Omega. Identical residues are marked with asterisk, whereas strongly and weakly similar residues are indicated with full stops and 
colons, respectively. The cytochrome $b 5$-like domain is underlined with a dotted line and the four putative transmembrane domains are underlined with solid lines. The heme-binding motif (HPGG) is highlighted in blue. The three conserved histidine boxes ( $\mathrm{XXXXH}, \mathrm{HXXHH}$ and QXXHH) are illustrated and highlighted with green, whereas regions involved in substrate specificity and regioselectivity are highlighted in yellow
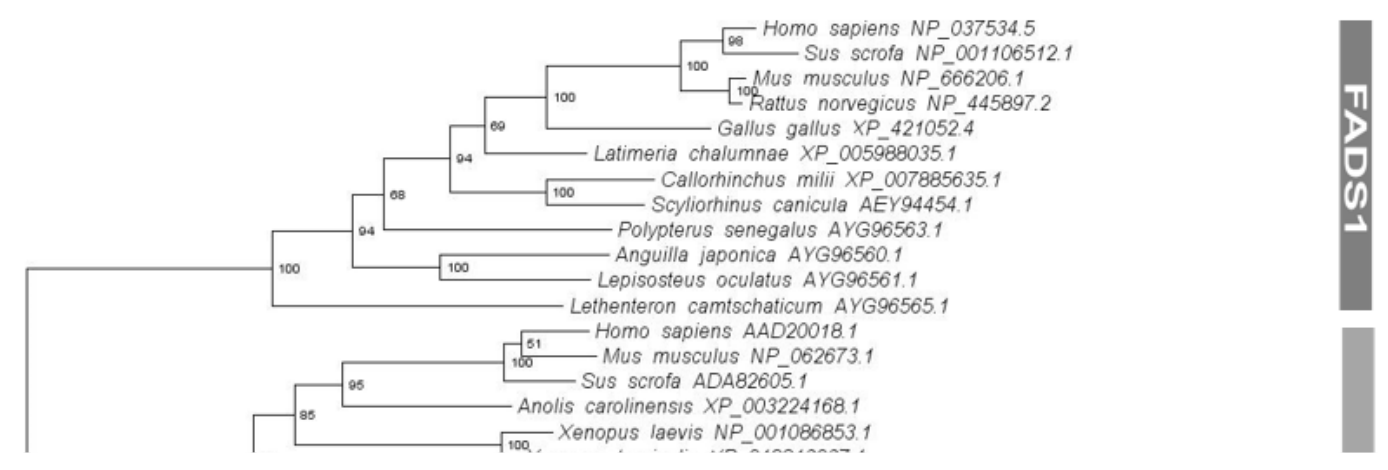

Figure 2 
Maximum likelihood phylogenetic analysis of the deduced amino acid sequence of the $P$. micromegethes Fads2 with Fads1 and Fads2 orthologs from other vertebrate species rooted with invertebrate group. Node values indicate bootstrap values. The $P$. micromegethes sequence is indicated in bold

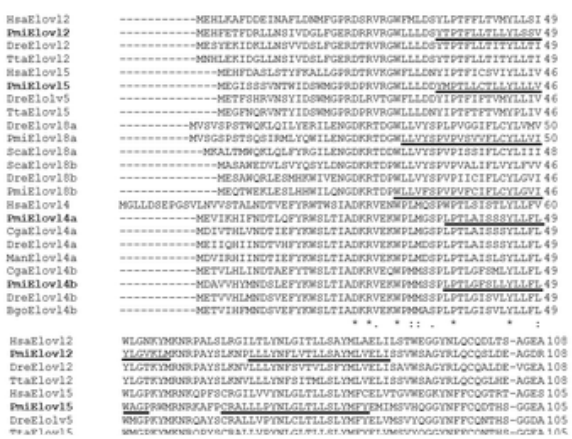

\section{Figure 3}


Comparison of the deduced amino acid (aa) sequence of the newly cloned elongases (Elovls) of the $P$. micromegethes (Elov15-UBB58396, Elovl2-UBB58397, Elov14a-UBB58398, and Elovl4b- UBB58399) and orthologues from Homo sapiens (Elovl5-NP_068586, Elovl2-NP_060240, and Elovl4-NP_073563), Clarias gariepinus (Elovl4a-ASY01350 and Elovl4b-ASY01351), Danio rerio (Elovl5-AAN77156, Elovl2NP_001035452, Elovl4a-NP_957090, Elovl4b-NP_956266, Elovl8a-QBZ96188, and Elovl8bNP_001191453), Barbonymus gonionotus (Elov15-AXG50646, Elovl2-AZN23179, Elovl4-QEE04393), Misgurnus anguillicaudatus (Elovl4a-QFR04622), Siganus canaliculatus (Elovl8a-QMU95576 and Elovl8bQMU95577), and Tor tambroides (Elovl5-QJU12162 and Elovl2-QJU12161) through alignment using Clustal Omega. Identical residues are marked with asterisk, whereas strongly and weakly similar residues are indicated with full stops and colons, respectively. The four conserved motifs (KXXEXXDT, QXXFLHXXHH, NXXXHXXMYXYY, and TXXQXX) are illustrated and highlighted with green, whereas the seven putative transmembrane domains are underlined with bold lines. The conserved histidine box $(H X X H H)$ is solid black framed. The putative endoplasmic reticulum (ER) retrieval signal located at the carboxyl terminus are highlighted in yellow

\section{Figure 4}

Phylogenetic tree inferred from deduced amino acid sequence of the P. micromegethes Elovl5, Elovl2, Elovl4a, and Elovl4b with orthologs from other vertebrate species using maximum likelihood analysis. Node values indicate bootstrap values. The $P$. micromegethes sequences are indicated in bold 


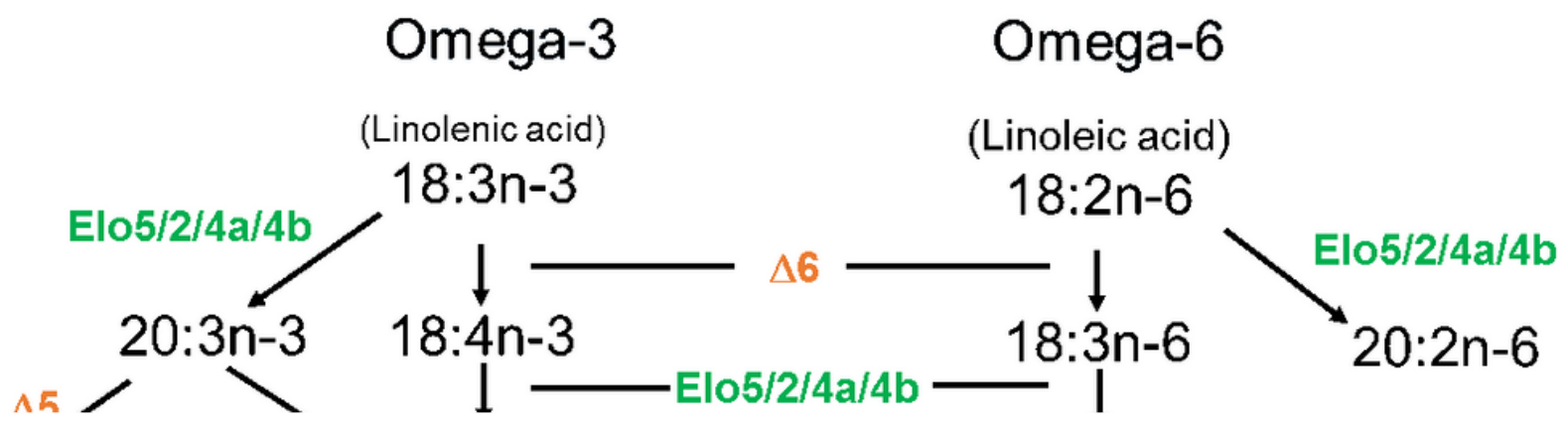

\section{Figure 5}

Proposed biosynthetic pathways of LC-PUFA (C20-24) and VLC-PUFA (>C24) from linolenic (18:3n-3) and linoleic (18:2n-6) acids in P. micromegethes by in vitro characterization of Fads2 desaturase ('Dx' coloured in orange) and Elovl elongases ('Elox' coloured in green) capacities using yeast. Elongation reactions of VLC-PUFA catalyzed by Elovl4 enzymes are indicated using blue arrow. 'Sprecher' pathway involved in the biosynthesis of docosahexaenoic acid (DHA 22:6n-3) from 22:5n-3 is highlighted with yellow background

\section{Supplementary Files}

This is a list of supplementary files associated with this preprint. Click to download.

- 2022PaedocyprisLCPUFAFPBSupplementary.docx 